\title{
Bright light therapy in the treatment of childhood and adolescence depression, antepartum depression, and eating disorders
}

\author{
Krzysztof Krysta $\cdot$ Marek Krzystanek • \\ Małgorzata Janas-Kozik • Irena Krupka-Matuszczyk
}

Received: 28 January 2012/ Accepted: 30 June 2012/Published online: 19 July 2012

(c) The Author(s) 2012. This article is published with open access at Springerlink.com

\begin{abstract}
Circadian rhythm disorders represent an important component underlying the pathology of depression. One of the subtypes of depression, in which these disorders may play a crucial role, is the seasonal affective disorder (SAD). The bright light therapy (BLT) has been reported as a novel, promising treatment method for SAD since 1984, and most of the data revealing its efficacy has been referred to adult patients, without comorbid disorders. However, in the recent years, more and more reports have been presented, which confirm the usefulness of BLT in some specific subpopulations of patients, including children, adolescents and pregnant women. The present review summarizes the applications of BLT in antepartum depression, childhood and adolescence depression as well as in patients suffering from eating disorders. Although the body of evidence is still too small to recommend the use of BLT as the first line of treatment for the depression or eating disorders in these patient subpopulations; it appears that BLT may be a useful alternative or adjunctive therapy for these diseases. However, the specific clinical applications of BLT in these areas need further investigation.
\end{abstract}

Keywords Bright light therapy (BLT) - Antepartum depression - Depression of childhood and adolescence . Eating disorders

\footnotetext{
K. Krysta ( $\square)$ - M. Krzystanek · I. Krupka-Matuszczyk Department of Psychiatry and Psychotherapy, Medical University of Silesia, Ziołowa 45/47, 40-635 Katowice, Poland

e-mail: krysta@mp.pl

M. Janas-Kozik

Department of Child and Adolescent Psychiatry and Psychotherapy, Medical University of Silesia,

Zapolskiej 3, 41-218 Sosnowiec, Poland
}

\section{Introduction}

A possible connection between the psychopathology of depressive disorders and circadian rhythms has been postulated for many years; however, the underlying biological mechanisms still remain unexplained. There are some data suggesting that the effect of antidepressive medications is at least partially due to their action on the circadian rhythms (McClung 2007). Exploring of the neurobiological mechanisms has contributed to the discovery of so-called clock genes, which are present in many cells of the central nervous system, and which are responsible for the circadian rhythm abnormalities, leading to disrupted behavioral states (Turek 2007). These biological connections may explain how close are the relations between sleep disorders and depressive symptoms. According to Harvey (2011), sleep disturbances are important, but still under-recognized mechanisms, related to the etiology and clinical course of mood disorders. In particular, abnormal sleep patterns may be risk factors for the relapse of depressive episodes as well as for the deterioration of cognitive and emotional regulation, and hormonal or metabolic disorders.

According to Antonijevic (2008), there may be different subtypes of depression, due to some alterations in functioning of the hypothalamic-pituitary-adrenal (HPA) axis, and different patterns of sleep architecture. This kind of distinction may serve as a future marker, potentially helpful in the selection of individually targeted treatment strategies. One of the first subtypes of depression, in which the positive effects of bright light therapy (BLT) were observed, was the seasonal affective disorder (SAD). Rosenthal et al. (1984) reported the first attempt of using BLT in a group of 29 patients, who were diagnosed with the bipolar disorder. Since that time, a number of studies analyzing the efficacy of BLT have been published. The 
mechanism of BLT is complex and different explanations have been proposed, including the circadian rhythm hypothesis, based on the sleep phase shift. This might mean that the individuals suffering from SAD during the winter might have the sleep phase delayed or advanced, in relation to their sleep/wake cycle (Lewy et al. 2007). It is assumed that BLT applied in the morning reduces the secretion of melatonin in phase-shift delay. Depending on the individual circadian rhythm changes, the morning or evening BLT application could be indicated as adjunctive chronobiological treatment methods for SAD.

\section{Biological mechanisms of the BLT action}

One of the most important anatomical structures, responsible for mood control is the suprachiasmatic nucleus ( $\mathrm{SCN}$ ), which is considered to be the circadian pacemaker of many different physiological and behavioral processes (Arushanian and Popov 2011). The neuronal activity of $\mathrm{SCN}$ is regulated by two G-protein coupled melatonin receptors, MT1 and MT2, and by melatonin secretion from the pineal gland. SCN receives neuronal impulses from the retina via the retinohypothalamic tract (Dubocovich 2007). Therefore, the level of melatonin secretion depends on the exposure to light during the day time, and also, on the season of the year. The exact way of the SCN regulation still remains hypothetical. One possibility is that the indolamines (melatonin and its precursor serotonin) have an ability to transduce light signals into the SCN. Another explanation is that the serotonin may play some role in the modulation of SCN networks, and in melatonin production by the pineal gland that influences biological rhythms.

Moreover, it has been hypothesized that the light exerts neurotrophic influence on neurons, and thus, a prolonged light deprivation can cause functional impairments of the noradrenergic-locus coeruleus system. In their experimental work, Gonzalez and Aston-Jones (2008) showed that the permanent light deprivation in rats increased apoptosis in the locus coeruleus; however, this effect can be reversed by some antidepressant drugs.

Also, there are some studies suggesting that the mechanism of antidepressant effect of BLT is connected with the functional augmentation of serotonergic and noradrenergic network systems. As an illustration, a tryptophan-free diet, and an administration of the hydroxylase inhibitor, alphamethyl-paratyrosine produce a robust increase of the depressive symptoms in patients diagnosed with SAD, while BLT administration remits these symptoms (Neumeister et al. 1998). It can be considered that the action of BLT is related to the augmentation of serotoninergic and noradrenergic transmission systems. In a study by Stastny et al. (2003), the serotoninergic and noradrenergic depletions in patients with SAD, who were in remission after receiving BLT, produced a transient reduction in plasma interleukine 4 levels. This may suggest that the BLT may influence the immune system regulation, which is often distorted in depression.

In addition, the alterations in neurohormones such as ghrelin and leptin may be involved in the pathophysiology of SAD. This can result in hyperphagia, with cravings for carbohydrates, and subsequently, can contribute to a moderate weight gain that frequently accompanies depressive and obsessive-compulsive disorders, which might favorably respond to BLT (Cizza et al. 2005; Garaulet et al. 2011).

According to the clinical observations by PraschakRieder and Willeit (2003), the morning BLT may be superior to the evening one, since it reduces the secretion of melatonin in phase-shift delay. The duration and frequency of BLT exposure are still controversial (Kronfeld-Schor and Einat 2012). For instance, Reeves et al. (2012) reported that a significant improvement in the depression scores can be found even after a single BLT session.

\section{Development of indications for BLT}

Golden et al. (2005) published a meta-analysis of studies reporting positive effects of BLT in affective disorders. The authors emphasized the existing differences in the methodology of the analyzed publications, including lack of rigorous study design. Nevertheless, in a majority of randomized, controlled studies, the beneficial effects of BLT in SAD and other depressive disorders were evident and comparable with the ones using antidepressants. This meta-analysis included only the studies, which enrolled adult patients (aged 18-65 years), and excluded the ones, which investigated children, adolescents and elderly patients. An interesting review was done by Pail et al. (2011), in which the authors paid a special attention to numerous obstacles that the researchers may encounter in designing their study protocols. The authors indicate that BLT is an efficacious, but probably underestimated treatment method for affective disorders, with good tolerability and acceptance.

Recently, some reports related to the BLT efficacy in depressive disorders among elderly patients have been found in the literature (Young 2011). Furthermore, the latest reports on the BLT clinical applications have revealed some new, potential indications, including antepartum depression, depression of childhood and adolescence. In these patient populations, a good tolerance of side effects is very important, and thus, novel, adjunctive forms of treatment are needed. 


\section{Antepartum depression}

The prevalence of antepartum depression differs according to the available sources, and can be estimated within the range 5-15 \% (Chatillon and Even 2010; Oren et al. 2002). According to Bader et al. (2010), about $11 \%$ of the pregnant women suffer from a major depressive disorder, which requires treatment. Under these circumstances, BLT can be one of the proposed therapeutic options, adjunctive to psychotherapy, antidepressants or electroconvulsive therapy (ECT). However, the literature reporting the results of non-pharmacological clinical trials in this area is still limited (Terman 2007; Golden et al. 2005). In their review of treatment methods used for the depression in pregnancy, Frisch and Riecher-Rössler (2010) and Ryan et al. (2005) proposed BLT as an alternative to antidepressants and psychotherapy. Similarly, Chatillon and Even (2010) reviewed the contemporary studies on antepartum depression and reported that the most widely used were tricyclic antidepressants and serotonin selective reuptake inhibitors (SSRIs), while the ECT was applied only in the most severe cases. They also documented that BLT seems to be effective; however, the studies are scarce and often inconclusive. In a study by Oren et al. (2002) in the group of 16 pregnant women, BLT was found to be effective in improving the Hamilton Depression Rating Scale (HDRS) scores by $49 \%$ after 3 weeks of treatment. Moreover, Epperson et al. (2004) reported successful treatment results of 10 pregnant women (enrolled in a randomized clinical trial) diagnosed with a major depressive disorder. In his review, Terman (2007) attempted to summarize the available research data concerning the application of BLT in pregnant women. However, he noted that in the past, this group of patients had been routinely excluded from the clinical trials, due to caution for the fetus. In one of the recent studies [a 5-year randomized, controlled clinical study by Wirz-Justice et al. (2011)], 27 pregnant women with non-seasonal major depressive disorder were treated with 7,000 lux bright light in the morning for $1 \mathrm{~h} /$ day, during a 5-week period. The authors reported that this 5-week BLT improved the depression in pregnancy, compared to placebo.

The treatment of depression during pregnancy is a demanding clinical challenge, which requires a consideration of the treatment risk, related to the fetus. Based on the above observations, BLT has been proposed as an alternative to antidepressant medication and was included into the recommendations of the Committee on Chronotherapeutics, delegated by the International Society for Affective Disorders (ISAD), for the use in patients who refuse, resist or cannot tolerate medication, or for whom medications may be contraindicated, as in a case of the antepartum depression (Wirz-Justice et al. 2005). It is worth emphasizing that the successful treatment of depression in pregnancy is related not only to clinical symptoms but also to some neurohormonal and endocrine patterns. Parry et al. (2006) measured the plasma levels of melatonin in pregnant women with depressive disorders and found that there was a lower mean melatonin amplitude, with lower mean evening cortisol levels, as compared to those levels in healthy controls. According to Parry and Newton (2001), the clinical improvement of antepartum depressive symptoms can be associated with the phase advances, of the melatonin rhythm. The above reports were also supported by our own clinical observation, describing the effects of depression treatment in three pregnant women (Krzystanek and Krupka-Matuszczyk 2006). These women were outpatients in 6, 7 and 8th-month of pregnancy, diagnosed with moderate depression, according to the ICD-10 criteria. Their treatment consisted of a morning exposure to 5,000 lux BLT for $1 \mathrm{~h}$, from Monday to Friday. Antidepressant effects were assessed after the 2nd and the 4th week of BLT. The mean improvement of depressive symptoms was 33 and $55 \%$ after 2 and 4 weeks of BLT, respectively. No side effects were observed in any of the patients. We have reported that the morning BLT is an effective and very well tolerated mode of treatment in pregnant women, suffering from non-seasonal depression. However, the exact technique and duration of BLT initiation and maintenance treatment require further studies. Antepartum depression is common in clinical practice, potentially endangering both the mother and her fetus. The adverse outcomes of antepartum depression include delivery complications and postpartum depression. In addition, there is an increased risk for preterm birth, low birth weight, and sudden death of the child (Chatillon and Even 2010). Therefore, the treatment requires an immediate and serious consideration and should not be postponed until the depression aggravates to more advanced stage.

\section{Depression of childhood and adolescence}

The literature reports some important differences between the treatment of depression in adults, and in children and adolescents. For example, some antidepressants effective in adult patients cause no improvement in the young patient population (Tsapakis et al. 2008). Although, this phenomenon has not been fully explained, one of the reasons for it may be the maturation of serotoninergic and noradrenergic systems (Bylund and Reed 2007). It is important to find out whether this type of differences might also be present in case of the BLT application. Some research trials, conducted in the $80 \mathrm{~s}$, were focused on the efficacy of BLT but were confined to the adult patients. The initial observations of the BLT application in children and adolescents were 
published in the $90 \mathrm{~s}$ by Sonis et al. (1987). Mghir and Vincent (1991) reported a case study of a 16-year-old girl with a 3-year history of SAD. After the phototherapy, a remission of her symptoms was observed. A few years later, the first analysis, referring to a larger group of patients, revealed the differences between SAD in adolescents and in adults (Glod et al. 1997), suggesting that the disregulated circadian rhythm patterns in children are comparable to those reported in non-seasonally depressed children; however, they are different from those observed in adults. This concept was further investigated in a study by Swedo et al. (1997), in which 28 children (aged 7-17 years) with pediatric SAD were enrolled in a doubleblind, placebo-controlled BLT trial. The total depression scores were significantly decreased during this treatment, indicating that BLT might be an effective treatment for pediatric SAD. However, these findings must be approached with caution, pending further studies in this area.

\section{Eating disorders}

The application of BLT has also been connected with some comorbidities in the adolescent patients, who may present not only depressive symptoms but also with other coexisting disorders. The rational explanation for the BLT use in eating disorders is its modulatory effect on the serotoninergic system functioning (Neumeister et al. 1998) which may be also deregulated in the eating disorders (Kaye et al. 2005). Symptoms of obsessive compulsive disorder (OCD) are common in both adults, and children and adolescents with anorexia nervosa (AN) (Hirani et al. 2010), and might be a part of the OCD-spectrum disorders (Altman and Shankman 2009). Based on this concept, a trial aimed at assessing the effects of 6-week BLT on depressive symptoms was conducted among 24 female patients with the restrictive type of anorexia nervosa (ANR) (Janas-Kozik et al. 2011). In this study, 12 subjects were randomly assigned to the BLT (10,000 lux, 30 min daily) intervention. The study patients did not suffer from depression prior to their AN onset. The depressive symptoms, observed in the study group, appeared to be secondary to the AN course. In addition, the depressive symptoms reported from this group of patients with AN-R did not have seasonal characteristics. These findings indicate that the improvement of depressive symptoms is significantly greater in the group receiving BLT. Moreover, a significant difference between the study groups (receiving BLT vs. control) with regard to the depression intensity scores after 5 and 6 weeks has been reported. The limitation of this study was its short observation period, and thus, in the future trials, a longer observation time would be needed to determine whether BLT may also be effective for preventing the relapse of depressive symptoms in AN-R patients. Despite these limitations, the results of this study suggested that BLT could be an effective non-pharmacological modality for the treatment of depression, in patients with AN-R.

This interesting issue of the symptom overlap between the depression and eating disorders was also observed in some earlier studies. In attempt of applying BLT in this type of comorbidity, Lam et al. (1994) described a group of 17 depressed patients with bulimia nervosa (BN), in whom it was found that BLT improved their symptoms, related both to the depression and eating disorder. However, the therapeutic effect of BLT in BN was greater in those patients, who suffered from a seasonal pattern. In a relevant case study by Ash et al. (1998), it was documented that the symptoms of eating disorder and depression had improved after 1 month of BLT. Furthermore, Daansen and Haffmans (2010) studied five women with chronic AN, who were treated with BLT, and reported that there is a shortterm positive effect of BLT on the anorexia symptoms. This finding suggests that the series of BLT procedures could be repeated after 3 months to augment the beneficial therapeutic effects.

In a double-blind, placebo-controlled study, Braun et al. (1999) described that BLT reduces binge frequency in 16 bulimic women, treated with 10,000 lux, in comparison with 18 patients receiving the dim red light (50 lux) therapy. However, there are also some negative results, published in the literature, concerning the improvement of BN symptoms after the BLT application (Blouin et al. 1996). In summary, the results of studies investigating the effects of BLT on AN and BN symptoms still remain controversial, and thus, more long-term studies are necessary to elucidate this alternative therapeutic possibility.

\section{Conclusion}

The BLT has been used as a treatment method for SAD. However, the most convincing evidence of its efficacy has been based on the studies referring to adult patients without comorbidities. Recently, the usefulness of BLT in some other psychiatric conditions has been illustrated in new therapeutic recommendations, including antepartum depression and depression of childhood and adolescence. Antepartum depression is a specific type of psychiatric disorder, in which a particular attention must be paid to the side effects of the treatment, especially for the fetus. Currently, BLT is a method considered to have minimal side effects for the mother and no known risk for the unborn child (Wirz-Justice et al. 2011).

In pregnant females, the rational use of pharmacotherapy or ECT was often limited due to possible adverse 
effects. Psychotherapy remained the safest method, although not always effective. Although more research is needed, the available results are fairly convincing that the BLT, as one of the treatment methods for antepartum and postpartum depression, presents a safe and effective alternative to pharmacotherapy (Golden et al. 2005).

As noted before, the early literature on the application of BLT in depression was limited to the adult population. After distinguishing certain differences between SAD in adults and in adolescents, the studies on BLT application confirmed its positive effects among children and adolescents (Sonis et al. 1987; Glod et al. 1997; Swedo et al. 1997).

In summary, the use of BLT in the depression of childhood and adolescence, and in the antepartum depression remains a promising alternative form of biological treatment, which is both effective and well tolerated. However, one should be aware of the fact that the body of evidence is still too small to create a strong recommendation for the use of BLT as the first line of treatment for depressive (childhood and adolescence and antepartum depression) or eating disorders ( $\mathrm{AN}$ and $\mathrm{BN}$ ), and future long-term studies on larger populations are necessary in this field. Overall, the existing research results are promising and should encourage investigators in their search in this area.

Conflict of interest The authors declare that they have no conflict of interest.

Open Access This article is distributed under the terms of the Creative Commons Attribution License which permits any use, distribution, and reproduction in any medium, provided the original author(s) and the source are credited.

\section{References}

Altman SE, Shankman SA (2009) What is the association between obsessive-compulsive disorder and eating disorders? Clin Psychol Rev 29:638-646

Antonijevic I (2008) HPA axis and sleep: identifying subtypes of major depression. Stress 11:15-27

Arushanian ÉB, Popov AV (2011) Recent data about the role of hypothalamic suprachiasmatic nucleus in circadian organization of physiological functions. Usp Fiziol Nauk 42:39-58

Ash JB, Piazza E, Anderson JL (1998) Light therapy in the clinical management of an eating-disordered adolescent with winter exacerbation. Int J Eat Disord 23:93-97

Bader A, Frisch U, Wirz-Justice A, Riecher-Rössler A (2010) Depression during pregnancy and its treatment. Nervenarzt 81:267-276

Blouin AG, Blouin JH, Iversen H, Carter J, Goldstein C, Goldfield G, Perez E (1996) Light therapy in bulimia nervosa: a double-blind, placebo-controlled study. Psychiatry Res 28:1-9

Braun DL, Sunday SR, Fornari VM, Halmi KA (1999) Bright light therapy decreases winter binge frequency in women with bulimia nervosa: a double-blind, placebo-controlled study. Compr Psychiatry 40:442-448

Bylund DB, Reed AL (2007) Childhood and adolescent depression: why do children and adults respond differently to antidepressant drugs? Neurochem Int 51:246-253

Chatillon O, Even C (2010) Antepartum depression: prevalence, diagnosis and treatment. Encephale 36:443-451

Cizza G, Romagni P, Lotsikas A, Lam G, Rosenthal NE, Chrousos GP (2005) Plasma leptin in men and women with seasonal affective disorder and in healthy matched controls. Horm Metab Res 37:45-48

Daansen PJ, Haffmans J (2010) Reducing symptoms in women with chronic anorexia nervosa. A pilot study on the effects of bright light therapy. Neuro Endocrinol Lett 31:290-296

Dubocovich ML (2007) Melatonin receptors: role on sleep and circadian rhythm regulation. Sleep Med 8(Suppl 3):34-42

Epperson CN, Terman M, Terman JS, Hanusa BH, Oren DA, Peindl KS, Wisner KL (2004) Randomized clinical trial of bright light therapy for antepartum depression: preliminary findings. J Clin Psychiatry 65:421-425

Frisch U, Riecher-Rössler A (2010) Depression during pregnancy. Ther Umsch 67:571-575

Garaulet M, Sánchez-Moreno C, Smith CE, Lee YC, Nicolás F, Ordovás JM (2011) Ghrelin, sleep reduction and evening preference: relationships to CLOCK 3111 T/C SNP and weight loss. PLoS One 28:e17435

Glod CA, Teicher MH, Polcari A, McGreenery CE, Ito Y (1997) Circadian rest-activity disturbances in children with seasonal affective disorder. J Am Acad Child Adolesc Psychiatry 36:188-195

Golden RN, Gaynes BN, Ekstrom RD, Hamer RM, Jacobsen FM, Suppes T, Wisner KL, Nemeroff CB (2005) The efficacy of light therapy in the treatment of mood disorders: a review and metaanalysis of the evidence. Am J Psychiatry 162:656-662

Gonzalez MM, Aston-Jones G (2008) Light deprivation damages monoamine neurons and produces a depressive behavioral phenotype in rats. Proc Natl Acad Sci USA 105:4898-4903

Harvey AG (2011) Sleep and circadian functioning: critical mechanisms in the mood disorders? Annu Rev Clin Psychol 7:297-319

Hirani V, Serpell L, Willoughby K, Neiderman M, Lask B (2010) Typology of obsessive-compulsive symptoms in children and adolescents with anorexia nervosa. Eat Weight Disord 15:86-89

Janas-Kozik M, Krzystanek M, Stachowicz M, Krupka-Matuszczyk I, Janas A, Rybakowski JK (2011) Bright light treatment of depressive symptoms in patients with restrictive type of anorexia nervosa. J Affect Disord 130:462-465

Kaye WH, Frank GK, Bailer UF, Henry SE (2005) Neurobiology of anorexia nervosa: clinical implications of alterations of the function of serotonin and other neuronal systems. Int $\mathrm{J}$ Eat Disord 37:S20-S21

Kronfeld-Schor N, Einat H (2012) Circadian rhythms and depression: human psychopathology and animal models. Neuropharmacology 62:101-114

Krzystanek M, Krupka-Matuszczyk I (2006) Bright light therapy in pregnant women depression-3 case studies. Psychiatr Pol 40:261-267

Lam RW, Goldner EM, Solyom L, Remick RA (1994) A controlled study of light therapy for bulimia nervosa. Am J Psychiatry 15:744-750

Lewy AJ, Rough JN, Songer JB, Mishra N, Yuhas K, Emens JS (2007) The phase shift hypothesis for the circadian component of winter depression. Dialogues Clin Neurosci 9:291-300

McClung CA (2007) Circadian genes, rhythms and the biology of mood disorders. Pharmacol Ther 114:222-232

Mghir R, Vincent J (1991) Phototherapy of seasonal affective disorder in an adolescent female. J Am Acad Child Adolesc Psychiatry 30:440-442 
Neumeister A, Turner EH, Matthews JM, Postolache TT, Barnett RL, Rauh M, Vetticad RG, Kasper S, Rosenthal NE (1998) Effects of tryptophan depletion vs catecholamine depletion in patients with seasonal affective disorder in remission with light therapy. Arch Gen Psychiatry 55:524-530

Oren DA, Wisner KL, Spinelli M, Epperson CN, Peindl KS, Terman JS, Terman M (2002) An open trial of morning light therapy for treatment of antepartum depression. Am J Psychiatry 159:666-669

Pail G, Huf W, Pjrek E, Winkler D, Willeit M, Praschak-Rieder N, Kasper S (2011) Bright-light therapy in the treatment of mood disorders. Neuropsychobiology 64:152-162

Parry BL, Newton RP (2001) Chronobiological basis of femalespecific mood disorders. Neuropsychopharmacology 25:102-108

Parry BL, Martínez LF, Maurer EL, López AM, Sorenson D, Meliska CJ (2006) Sleep, rhythms and women's mood. Part I. Menstrual cycle, pregnancy and postpartum. Sleep Med Rev 10:129-144

Praschak-Rieder N, Willeit M (2003) Treatment of seasonal affective disorders. Dialogues Clin Neurosci 5:389-398

Reeves GM, Nijjar GV, Langenberg P, Johnson MA, Khabazghazvini B, Sleemi A, Vaswani D, Lapidus M, Manalai P, Tariq M, Acharya M, Cabassa J, Snitker S, Postolache TT (2012) Improvement in depression scores after 1 hour of light therapy treatment in patients with seasonal affective disorder. J Nerv Ment Dis 200:51-55

Rosenthal NE, Sack DA, Gillin JC, Lewy AJ, Goodwin FK, Davenport Y, Mueller PS, Newsome DA, Wehr TA (1984) Seasonal affective disorder. A description of the syndrome and preliminary findings with light therapy. Arch Gen Psychiatry 41:72-80

Ryan D, Milis L, Misri N (2005) Depression during pregnancy. Can Fam Physician 10:1087-1093
Sonis WA, Yellin AM, Garfinkel BD, Hoberman HH (1987) The antidepressant effect of light in seasonal affective disorder of childhood and adolescence. Psychopharmacol Bull 23:360-363

Stastny J, Konstantinidis A, Schwarz MJ, Rosenthal NE, Vitouch O, Kasper S, Neumeister A (2003) Effects of tryptophan depletion and catecholamine depletion on immune parameters in patients with seasonal affective disorder in remission with light therapy. Biol Psychiatry 53:332-337

Swedo SE, Allen AJ, Glod CA, Clark CH, Teicher MH, Richter D, Hoffman C, Hamburger SD, Dow S, Brown C, Rosenthal NE (1997) A controlled trial of light therapy for the treatment of pediatric seasonal affective disorder. J Am Acad Child Adolesc Psychiatry 36:816-821

Terman M (2007) Evolving applications of light therapy. Sleep Med Rev 11:497-507

Tsapakis EM, Soldani F, Tondo L, Baldessarini RJ (2008) Efficacy of antidepressants in juvenile depression: meta-analysis. $\mathrm{Br} \mathrm{J}$ Psychiatry 193:10-17

Turek FW (2007) From circadian rhythms to clock genes in depression. Int Clin Psychopharmacol 22(Suppl 2):S1-S8

Wirz-Justice A, Benedetti F, Berger M, Lam RW, Martiny K, Terman M, Wu JC (2005) Chronotherapeutics (light and wake therapy) in affective disorders. Psychol Med 35:939-944

Wirz-Justice A, Bader A, Frisch U, Stieglitz RD, Alder J, Bitzer J, Hösli I, Jazbec S, Benedetti F, Terman M, Wisner KL, RiecherRössler A (2011) A randomized, double-blind, placebo-controlled study of light therapy for antepartum depression. J Clin Psychiatry 72:986-993

Young SN (2011) Bright light for nonseasonal depression? J Psychiatry Neurosci 2011(36):E37-E38 